\title{
Effect of disorder on 2D topological merging transition from a Dirac semi-metal to a normal insulator
}

\author{
David Carpentier, Andrei A. Fedorenko and Edmond Orignac \\ CNRS UMR5672 - Laboratoire de Physique de l'Ecole Normale Supérieure de Lyon, \\ '46, Allée d'Italie, 69007 Lyon, France
}

PACS 73.20.At - Surface states, band structure, electron density of states PACS $73.20 . \mathrm{Hb}$ - Impurity and defect levels; energy states of adsorbed species PACS 73.43.Nq - Quantum phase transitions

\begin{abstract}
We study the influence of disorder on the topological transition from a twodimensional Dirac semi-metal to an insulating state. This transition is described as a continuous merging of two Dirac points leading to a semi-Dirac spectrum at the critical point. The latter is characterized by a dispersion relation linear in one direction and quadratic in the orthogonal one. Using the self-consistent Born approximation and renormalization group we calculate the density of states above, below and in the vicinity of the transition in the presence of different types of disorder. Beyond the expected disorder smearing of the transition we find an intermediate disordered semi-Dirac phase. On one side this phase is separated from the insulating state by a continuous transition while on the other side it evolves through a crossover to the disordered Dirac phase.
\end{abstract}

Introduction. - In the recent years there has been a growing interest for materials whose low energy degrees 'of freedom are described by two dimensional (2D) Dirac fermions. For instance, 2D Dirac points have been found in the energy spectrum of graphene 1,2, 3D topological insulators 3, 4, such as the materials in the $\mathrm{Bi}_{2} \mathrm{Se}_{3}$ fam'ily [5] and strained HgTe [6], quasi-2D organic conductor $\alpha$-(BEDT-TTF $)_{2} \mathrm{I}_{3}$ under pressure $7-10$ and very tunable molecular system assembled by atomic manipulation of carbon monoxide molecules on a copper surface 11. 'Each Dirac point carries a topological charge which is robust against perturbations [12. This topological charge is related to the chirality of the electronic states, but can also 'be viewed as a Berry monopole. The Berry phase associated with any closed path in reciprocal space is determined by the total topological charge of the Dirac points enclosed by the path. Moreover, in the case of two-dimensional models, excluding the surface states, according to the fermion doubling theorem the Dirac cones emerge by pairs of opposite topological charges. In this case, a topological transition towards an insulator is possible which consists of a merging of the Dirac points of opposite topological charges 13 . Moving the Dirac points in the Brillouin zone up to their fusion requires perturbations of the underlying microscopic Hamiltonian. This can be achieved, for exam- ple, by mechanical stretching or depositing adatoms that could give rise to a hopping anisotropy or enhancement of higher-order hopping amplitudes. Exactly at the topological transition triggered by the merging of two Dirac points, a very peculiar semi-Dirac dispersion relation occurs, which is linear in one direction while being quadratic in the orthogonal one 13 17]. Recently such a merging of tunable Dirac points has been realized using a degenerate Fermi gas trapped in a 2D honeycomb optical lattice [18, in photonic graphene [19] and in microwave experiments 20]. Moving and merging of two Dirac points in the organic conductor $\alpha$-(BEDT-TTF $)_{2} \mathrm{I}_{3}$ has also been discussed in [7,21,22].

For 2D Dirac semi-metals, both the density of states (DOS) near the Dirac point and the transport properties may be strongly affected by the presence of disorder. Its effect crucially depends on properties of disorder such as preserved symmetries or the range of correlations. This has been intensively studied using different methods during last two decades 23 30]. On the contrary, much less is known on the disorder effects close to and at the topological semi-metal-insulator transition. Very recently, the Friedel oscillations induced in the semi-Dirac fermions by a single localized impurity have been studied in 31. In the present paper we address the effect of multiple randomly 

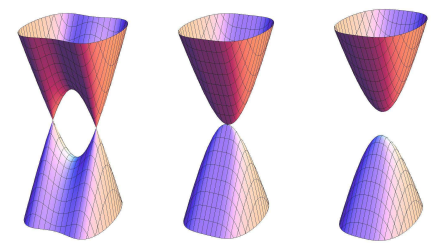

Fig. 1: The energy spectrum of the clean system: The left panel corresponds to $\Delta<0$, the right panel to $\Delta>0$. The center panel is the semi-Dirac point $\Delta=0$.

distributed impurities of different types on the DOS in the vicinity of the topological transition. We focus on the DOS at low energy, near the original Dirac points, while the different problem of diffusion of semi-Dirac fermions at higher Fermi energy will be presented elsewhere [32.

Model. - We consider a 2D semi-metal with valence and conduction bands touching each other at two Dirac points. We assume that the Dirac points can evolve in reciprocal space upon variation of the parameter $\Delta$ which drives a topological Lifshitz transition [33]: for the critical value $\Delta=0$ the two points merge as shown in fig. 1. The effective Hamiltonian describing the low energy physics in the vicinity of the transition can be derived from the tightbinding model expanding around the merging point. It is expected to be general and reads 13 .

$$
H_{0}=\sigma_{x}\left(\Delta-\frac{\partial_{x}^{2}}{2 m}\right)-i c \sigma_{y} \partial_{y} .
$$

At the critical point $\Delta=0$, Hamiltonian (1) describes the semi-Dirac fermions with a quadratic dispersion relation parametrized by the mass $m$ in the $x$-direction, and linear dispersion with velocity $c$ in the $y$-direction. We assume that $m>0$ and set $\hbar=1$, while $\sigma_{0}=\mathbb{I}$ and the three $\sigma_{\mu}$, $\mu=x, y, z$ are the Pauli matrices. The diagonalization of Hamiltonian (11) via Fourier transform gives the energy spectrum $\varepsilon= \pm\left[\left(\Delta+q_{x}^{2} / 2 m\right)^{2}+c^{2} q_{y}^{2}\right]^{1 / 2}$. Note that in the Hamiltonian (11), the $x$ direction corresponding to the real $\sigma_{x}$ matrix, has been identified with the direction connecting the two cones for $\Delta<0$. With this convention, the model is clearly invariant under time reversal symmetry for any value of $\Delta$. This is indeed the situation which arises on any tight-binding lattice model. Exchanging the $x$ and $y$ directions leads to a model which breaks time reversal symmetry, and describes a different physics.

To incorporate in the model the presence of randomly distributed impurities we add to Hamiltonian (11) a random perturbation which can be parametrized in full generality by the four disorder potentials $V_{i}(\mathbf{r})$ :

$$
H=H_{0}+\sum_{i=0, x, y, z} \sigma_{i} V_{i}(\mathbf{r}) .
$$

The potentials $V_{i}(\mathbf{r})$ are assumed to be random Gaussian fields with zero mean $\overline{V_{i}(\mathbf{r})}=0$. The disorder strengths

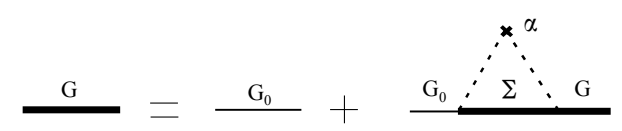

Fig. 2: The averaged over disorder Green function in the SCBA.

$\alpha_{i}$ parametrize the variance of the distribution according to

$$
\overline{V_{i}(\mathbf{r}) V_{j}\left(\mathbf{r}^{\prime}\right)}=\frac{2 \pi c \alpha_{i}}{\sqrt{m}} \delta_{i j} \delta\left(\mathbf{r}-\mathbf{r}^{\prime}\right) .
$$

All these terms have simple physical meanings: $V_{0}(\mathbf{r})$ is a random scalar potential, $V_{x}(\mathbf{r})$ describes local fluctuations of $\Delta, V_{y}(\mathbf{r})$ is a random gauge potential acting in the $y$ directions, while $V_{z}(\mathbf{r})$ plays the role of the random Dirac mass. The system (2) resembles mathematically the Lifshitz point in anisotropic magnets where a disordered, an ordered and a spatially modulated phase meet [35].

The self-consistent Born approximation . - To describe the effect of disorder on the DOS at low energy, we naturally employ the self consistent Born approximation (SCBA). The SCBA is expected to be reliable, except possibly in the very vicinity of the transition 27,34 . The retarded and advanced Green functions for the Hamiltonian without disorder (11) written in Fourier space are given by

$$
G_{0}^{R / A}(\varepsilon, \mathbf{q})=\frac{\varepsilon+\left(\Delta+\frac{q_{x}^{2}}{2 m}\right) \sigma_{x}+c q_{y} \sigma_{y}}{(\varepsilon \pm 0)^{2}-\left(\Delta+\frac{q_{x}^{2}}{2 m}\right)^{2}-c^{2} q_{y}^{2}} .
$$

To calculate the corresponding Green functions averaged over disorder within the SCBA one takes into account the one-loop diagram contributing to the self-energy. Replacing the bare Green function in this diagram by the dressed one, we obtain a self-consistent equation for the self-energy $\Sigma(\varepsilon)$ graphically depicted in fig. 2. The equation is simplified by neglecting the external momenta dependance of the self-energy $\Sigma(\varepsilon)$. For convenience we introduce the matrix function $X(\varepsilon)=\varepsilon-\Sigma(\varepsilon)$. Making an ansatz $X(\varepsilon)=X_{0}(\varepsilon) \sigma_{0}-X_{1}(\varepsilon) \sigma_{x}$, where $X_{i}(\varepsilon)$ are scalar functions, we can rewrite the SCBA equation as

$$
\begin{aligned}
& X_{0}(\varepsilon)=\varepsilon+\frac{2 \pi c \alpha}{\sqrt{m}} \int_{q} \frac{X_{0}(\varepsilon)}{\Omega\left[X_{1}, q_{x}\right]^{2}+c^{2} q_{y}^{2}-X_{0}^{2}(\varepsilon)}, \\
& X_{1}(\varepsilon)=-\frac{2 \pi c \tilde{\alpha}}{\sqrt{m}} \int_{q} \frac{\Omega\left[X_{1}, q_{x}\right]}{\Omega\left[X_{1}, q_{x}\right]^{2}+c^{2} q_{y}^{2}-X_{0}^{2}(\varepsilon)} .
\end{aligned}
$$

Here we have denoted $\int_{q}:=\int d^{2} q /(2 \pi)^{2}$ and $\Omega\left[X_{1}, q_{x}\right]:=$ $\Delta+X_{1}(\varepsilon)+q_{x}^{2} /(2 m)$, and also defined the total disorder strength $\alpha:=\alpha_{0}+\alpha_{x}+\alpha_{y}+\alpha_{z}$ and the disorder asymmetry $\tilde{\alpha}:=\alpha_{0}+\alpha_{x}-\alpha_{y}-\alpha_{z}$. We first notice that the UV diverging integral in eq. (5b) is determined by the cutoff $\Lambda$. To simplify calculations we impose the cutoff only on wavevectors in the $x$-direction, so that $\left|q_{x}\right|<\Lambda$, while we will neglect it in the $y$-direction. Integrating over $q_{x}$ and 


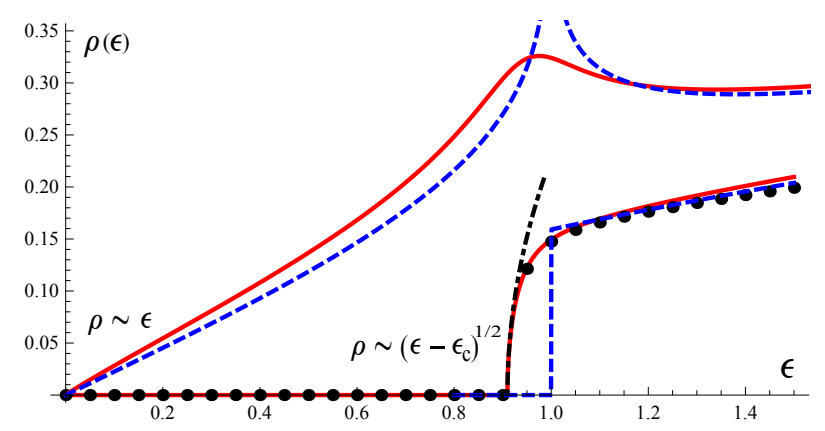

Fig. 3: The DOS in units of $\sqrt{m} / c$ deep in the insulating phase for $\bar{\Delta}=1$ (bottom plot) and in the semi-metal phase for $\bar{\Delta}=-1$ (upper plot). The disorder strength is $\alpha=0.02$. In the insulating phase the DOS vanishes for $\varepsilon<\varepsilon_{c} \approx 0.91$. The red solid lines are obtained using numerical solution of eqs. (8) and (12); blue dashed lines using the first iteration of the latter equations that gives the shifted DOS of the clean system; black dots using numerical solution of the simplified eq. (9). The black dot-dashed line is the power-law approximation (11).

$q_{y}$ in eq. (5b) we obtain $X_{1}=-\tilde{\alpha} \Lambda / \sqrt{m}$ for large $\Lambda$ which does not depend on $\varepsilon$. It is convenient to introduce the renormalized driving parameter

$$
\bar{\Delta}=\Delta-\frac{\tilde{\alpha}}{\sqrt{m}} \Lambda
$$

which accounts for the shift due to disorder asymmetry of the semi-metal to insulator transition point from $\Delta=0$ towards $\bar{\Delta}=0$. The shift depends on the cutoff and thus is non universal similar to what happens at the classical Lifshitz point [35. Solving eq. (5a) for $X_{0}$ we find the DOS from the expression

$$
\rho(\varepsilon)=-\frac{1}{\pi} \operatorname{Im} \operatorname{Tr} \int_{q} G^{R}(\varepsilon, \mathbf{q})=\frac{\sqrt{m}}{\alpha c \pi^{2}} \operatorname{Im} X_{0}^{R}(\varepsilon) .
$$

The form of the integral over $\mathbf{q}$ in eq. (5a) strongly depends on the sign of $\bar{\Delta}$, i.e. on the direction from which we approach the topological transition. Hence we first discuss the effect of disorder deep in the insulating $(\bar{\Delta}>0)$ and semi-metal $(\bar{\Delta}<0)$ phases, and finally we concentrate on the vicinity of the topological transition $(\bar{\Delta} \approx 0)$.

The insulating phase. For $\bar{\Delta} \geq 0$, integrating over $\mathbf{q}$ in eq. (5a) we obtain

$$
X_{0}=\varepsilon+\frac{\sqrt{2} \alpha X_{0}}{\sqrt{\bar{\Delta}-X_{0}}} K\left(2+\frac{2 \bar{\Delta}}{X_{0}-\bar{\Delta}}\right),
$$

where $K(z)=\int_{0}^{1} d t\left[\left(1-t^{2}\right)\left(1-z t^{2}\right)\right]^{-1 / 2}$ is the complete elliptic integral of the first kind 1 Equation (8) has two complex conjugate solutions corresponding to the retarded and advanced functions. We always find for $\bar{\Delta}>\bar{\Delta}_{c}=\pi^{2} \alpha^{2} / 2$ the DOS of an insulator: there exists an energy gap $\varepsilon_{c}=\bar{\Delta}+O(\alpha)$ such that the solution

${ }^{1}$ Note that this definition differs from that used in 13 by changing $K(z) \rightarrow K(\sqrt{z})$. of eq. (8) is purely real for $0 \leq \varepsilon \leq \varepsilon_{c}$, and thus, the DOS $\rho(\varepsilon)$ vanishes. The DOS computed using eq. (7) and the imaginary part of the numerical solution of eq. (8) is shown in fig. 3. For $\left|\varepsilon-\varepsilon_{c}\right| \gg 0$ one can solve eq. (8) by iterations: this provides an expansion of the solution in powers of disorder strength. The first iteration derived by taking $X_{0} \rightarrow \varepsilon+i 0^{+}$in the r.h.s of eq. (8) gives a linear in disorder correction and thus the DOS of the clean system found in [13] up to the shift $\Delta \rightarrow \bar{\Delta}$. It is also shown in fig. 3 for comparison. To study the solution of the SCBA equation in the vicinity of the energy gap $\varepsilon_{c}$, we replace the complete elliptic integral in eq. (8) by its asymptotics that yields

$$
X_{0}=\varepsilon+\frac{\alpha}{2} \sqrt{X_{0}}\left[\ln \left(\frac{32 X_{0}}{X_{0}-\bar{\Delta}}\right) \pm i \pi\right],
$$

where "+/-" correspond to the retarded and advanced functions, respectively. Equation (9) can be written as $X_{0}=\varepsilon+f\left(X_{0}\right)$ so that the energy gap $\varepsilon_{c}$ and the corresponding value $X_{0}\left(\varepsilon_{c}\right)=X_{c}$ satisfy: $X_{c}=\varepsilon_{c}+f\left(X_{c}\right)$ and $f^{\prime}\left(X_{c}\right)=1$. In the limit of weak disorder we obtain

$\varepsilon_{c}=\bar{\Delta}\left\{1-\frac{\alpha}{2 \sqrt{\bar{\Delta}}}\left[1-\ln \left(\frac{\alpha}{64 \sqrt{\bar{\Delta}}}\right)\right]\right\}+O\left(\alpha^{2} \ln \alpha\right)$,

and the expansion of the DOS around this point for $\varepsilon \gtrsim \varepsilon_{c}$ :

$$
\rho(\varepsilon)=\frac{\sqrt{m}}{\alpha c \pi^{2}}\left[\frac{2\left(\varepsilon-\varepsilon_{c}\right)}{f^{\prime \prime}\left(X_{c}\right)}\right]^{1 / 2}+O\left(\left(\varepsilon-\varepsilon_{c}\right)^{3 / 2}\right) .
$$

Thus, the DOS of the disordered system in the insulating phase vanishes as a power law with the exponent $\frac{1}{2}$ in contrast to the jump in the DOS of the clean system (see the bottom plot in fig. 3 ).

The semi-metallic phase. In the case $\bar{\Delta}<0$, the integration over $\mathbf{q}$ in eq. (5a) leads to the equation

$$
X_{0}=\varepsilon+\frac{\sqrt{2} \alpha X_{0}}{\sqrt{\bar{\Delta}+X_{0}}}\left[K\left(\frac{2 X_{0}}{\bar{\Delta}+X_{0}}\right)+2 i K\left(\frac{\bar{\Delta}-X_{0}}{\bar{\Delta}+X_{0}}\right)\right]
$$

The imaginary part of the numerical solution of eq. (12) gives the DOS which is shown in the upper plot of fig. 3 . Iterating eq. (12) one can obtain the solution for $|\varepsilon-\bar{\Delta}| \gg$ 0 . The DOS of the clean system found in 13. (up to the shift (6) $)$ is recovered by taking the limit $X_{0} \rightarrow \varepsilon+i 0^{+}$ in the r.h.s of eq. (12) and substituting the solution to eq. (17). This DOS diverges logarithmically at $\varepsilon=|\bar{\Delta}|$ while the DOS of the disordered system is continuous and smooth (see the upper plot in fig. 3).

Topological transition. Let us now focus on the region of small $\bar{\Delta}$. The DOS computed numerically using the SCBA eqs. (8) and (12) is shown in fig. 4 as a function of $\bar{\Delta}$ in the vicinity of the transition $\bar{\Delta}=0$. One can see that in the presence of disorder there is a region around the transition point $\bar{\Delta}=0$ characterized by a finite DOS at zero energy, $\rho(\varepsilon=0)>0$. The DOS at $\varepsilon=0$ strictly vanishes for $\bar{\Delta}>\bar{\Delta}_{c}=\pi^{2} \alpha^{2} / 2$. Thus, in the presence 

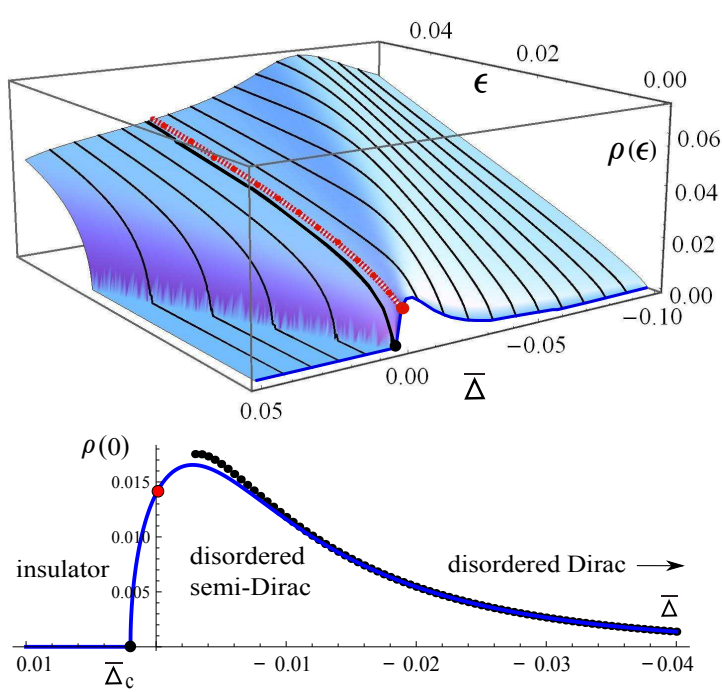

Fig. 4: The DOS in units of $\sqrt{m} / c$ close to the transition as a function of $\bar{\Delta}$ (upper panel at arbitrary energy, bottom panel at zero energy). The disorder strength is $\alpha=0.02$. The red dashed line is the DOS at $\bar{\Delta}=0$ given by eq. (15). The DOS at the critical point $\bar{\Delta}_{c} \approx 0.002$ is given by eq. (13). Dotted line in the bottom panel is given by eq. (14).

of disorder the transition (semi-Dirac) point between the Dirac semi-metal and the insulator is replaced by a new intermediate regime which we call the disordered semi-Dirac regime. The disordered Dirac phase smoothly evolves into this intermediate regime, while the SCBA suggests a sharp continuous transition at $\bar{\Delta}=\bar{\Delta}_{c}$ between this disordered semi-Dirac phase and the insulating phase. Exactly at the transition we find an algebraic behavior of the DOS

$$
\rho\left(\varepsilon ; \bar{\Delta}=\bar{\Delta}_{c}\right)=\left(\frac{3}{4}\right)^{1 / 6} \frac{\sqrt{m}}{c \pi}(\pi \alpha)^{1 / 3} \varepsilon^{1 / 3},
$$

which is displayed in fig. 4. The extrapolation towards the disordered semi-metallic regime is obtained for large negative $\bar{\Delta}<0$ : in this regime we recover a DOS at zero energy decaying as

$$
\rho(0)=\frac{8|\bar{\Delta}| \sqrt{m}}{\alpha c \pi^{2}} e^{-\sqrt{|\bar{\Delta}|} /(\alpha \sqrt{2})} .
$$

Hence, instead of a transition the system undergoes a crossover to disordered Dirac fermions. Indeed, this behavior is fully consistent with the SCBA picture for the disordered Dirac fermions. For graphene it predicts a finite $\operatorname{DOS} \rho(\varepsilon)=2 \Gamma_{0} /\left(\pi^{2} v_{0}^{2} \alpha_{D}\right)$ for $\varepsilon \ll \Gamma_{0}$ where $\Gamma_{0}=\Delta_{D} e^{-1 / \alpha_{D}}$ is the energy scale generated by disorder. Here $\Delta_{D}$ is the bandwidth, $v_{0}$ - the Fermi velocity and $\alpha_{D}$ - the dimensionless strength of disorder [27, 28. This would imply a finite DOS at zero energy for all considered types of disorder. However, this contradicts the results obtained for random gauge and random mass disorder using more reliable methods. For instance, for random gauge disorder one expects $\rho(\varepsilon)=\varepsilon^{2 / z-1}$ with $z=1+\alpha_{D}$ in weak disorder case $\left(\alpha_{D}<2\right)\left[23\right.$ and $z=\left(8 \alpha_{D}\right)^{1 / 2}-1$ in strong disorder case $\left(\alpha_{D}>2\right)$ [25]. Similarly, with random mass disorder, the DOS remains linear in $\varepsilon$ up to logarithmic corrections [30]. Only for scalar potential disorder the DOS saturates at a finite value in the vicinity of the Dirac point 27. It was argued in 24 that the failure of SCBA in the vicinity of the Dirac point for $\varepsilon<\Gamma_{0}$ is due to importance of the diagrams with crossed disorder lines, neglected within the SCBA which takes into account only the non-crossed ones. Another possible reason for the failure of the SCBA is the divergence of the fermion wavelengths at the Dirac point rendering the SCBA uncontrollable, i.e. without a small parameter. Nevertheless one can still rely on the SCBA for energies $\varepsilon \gg \Gamma_{0}$. The question of reliability of the SCBA in the vicinity of the topological transition is even more subtle.

Disordered Semi-Dirac. It is interesting to note that the SCBA equations can be analytically solved exactly at the point $\bar{\Delta}=0$, inside the "disordered semi-Dirac" regime. Indeed using that $K\left(2+0^{+} / z\right)=C(1-$ $\operatorname{sign}[\operatorname{Im} z] i) / \sqrt{2}$ where $C=\Gamma(1 / 4)^{2} /(4 \sqrt{\pi})$ one can rewrite eq. (8) as $X_{0}=\varepsilon+(1 \mp i) C \alpha \sqrt{-X_{0}}$, where "-/+" corresponds to the retarded/advanced Green's function. We obtain the DOS at the point $\bar{\Delta}=0$

$$
\rho(\varepsilon)=\frac{\sqrt{m} C^{2} \alpha}{c \pi^{2}}\left[1+\frac{1}{\sqrt{2}} \sqrt{1+\sqrt{1+\frac{4 \varepsilon^{2}}{C^{4} \alpha^{4}}}}\right],
$$

such that $\rho(0)=2 \sqrt{m} C^{2} \alpha /\left(c \pi^{2}\right)$. Note that the expansion of eq. (15) in powers of the disorder strength $\alpha$ is in fact an expansion in $\alpha / \sqrt{\varepsilon}$. Its truncation to a finite order in $\alpha$ appears to be pathological for $\varepsilon \ll \alpha^{2}$. We interpret this as a sign of the probable breakdown of the SCBA for a small enough energy. In this regime, a more refined technique is required 26.

Before we consider a weak disorder renormalization group (RG) approach to the topological transition let us first discuss the consequences of our SCBA results for the behavior of specific heat and spin magnetic susceptibility [13,36]. Assuming that the chemical potential is temperature independent and fixed at zero energy the specific heat can be written as eq. (18) in 13 . In the clean system the low $T$ specific heat interpolates from a $T^{2}$ behavior far in the semi-metal phase to an activated behavior in the insulating phase. Exactly at the transition it exhibits a $T^{3 / 2}$ behavior. In the presence of disorder the low $T$ specific heat at the critical point should behave as $T^{4 / 3}$. The magnetic susceptibility $\chi$ is related to the DOS by eq. (10.11) in [37. In the clean system, $\chi$ is activated in the insulator, $\chi \sim T$ in the semi-metal phase and $\chi \sim T^{1 / 2}$ at the transition. With disorder, the scaling behavior of the susceptibility at the transition is modified to $\chi \sim T^{1 / 3}$.

Weak disorder renormalization group. - We now study whether the critical point found using the SCBA is accessible within a weak disorder RG approach. The starting point is the generating functional for the Green functions $\mathcal{Z}=\int D \bar{\psi} D \psi e^{-\mathcal{S}}$, where $\mathcal{S}$ is the effective action 
at a given energy (Matsubara frequency) $\varepsilon$. The effective action can be written in terms of two fermionic (anticommuting) fields $\psi(\mathbf{r})$ and $\bar{\psi}(\mathbf{r})$ which are two completely independent Grassman variables. For generality we consider a class of $d$ dimensional models in which the bare dispersion is quadratic in $l$ direction and linear in $d-l$ remaining directions. For instance, the case $(d=2, l=1)$ describes the semi-Dirac fermions, $(d=3, l=0)$ - Weyl fermions 38 , and ( $d=3, l=1$ or 2$)$ - semi-Weyl fermions, e.g. similar to that found in ${ }^{3} \mathrm{He}-\mathrm{A}[15]$.

Scaling dimensions. The standard way to generalize the model to $d$ dimensions is to introduce the generators of the $d$-dimensional Clifford algebra. In even dimensions these generators can be represented by the matrices $\gamma_{i}(i=1, \ldots, d)$ of dimension $2^{d / 2}$. Generally one also defines $\gamma_{d+1} \equiv \gamma_{S}=i^{-d / 2} \gamma_{1} \ldots \gamma_{d}$. For the case of odd dimensions one constructs the matrices $\gamma_{i}$ $(i=1, \ldots, d-1)$ similarly to the case of even dimension and adds $\gamma_{d} \equiv \gamma_{S}=i^{-(d-1) / 2} \gamma_{1} \ldots \gamma_{d-1}$. These matrices satisfy the anticommutation relations: $\gamma_{i} \gamma_{j}+\gamma_{j} \gamma_{i}=2 \delta_{i j} \gamma_{0}$, $i, j=1, \ldots,[[d+1]]$, where $\gamma_{0}=\mathbb{I}$ is the identity matrix and $[[d+1]]$ stands for $d+1$ if $d$ is even, and $d$ if it is odd. The matrices $\gamma_{i}$ and $\gamma_{S}$ replace the Pauli matrices $\sigma_{i}(i=x, y)$ and $\sigma_{z}$ in $d$ dimensions. We define the two matrix vectors $\gamma_{\|}:=\left(\gamma_{1}, \ldots, \gamma_{l}\right)$ and $\gamma_{\perp}:=\left(\gamma_{l+1}, \ldots, \gamma_{d}\right)$ and replace in Hamiltonian (1) $\partial_{x} \rightarrow \partial_{\|}$which acts in $l$-dimensional subspace and $\partial_{y} \rightarrow \partial_{\perp}$ which acts in $(d-l)$ dimensional subspace. Introducing $n$ copies of the original system and using the replica trick to average over disorder we can write the effective replicated action as

$$
\begin{aligned}
& \mathcal{S}=\int d^{d} \mathbf{r}\left\{\sum_{a=1}^{n} \bar{\psi}_{a}\left[\gamma_{\|}\left(\Delta-\frac{\partial_{\|}^{2}}{2 m}\right)-i c \gamma_{\perp} \partial_{\perp}-i \varepsilon\right] \psi_{a}\right. \\
& \left.-\frac{\pi c}{\sqrt{m}} \sum_{a, b=1}^{n} \sum_{j=0}^{[[d+1]]} \alpha_{j}\left[\bar{\psi}_{a}(\mathbf{r}) \gamma_{j} \psi_{a}(\mathbf{r})\right]\left[\bar{\psi}_{b}(\mathbf{r}) \gamma_{j} \psi_{b}(\mathbf{r})\right]\right\},
\end{aligned}
$$

where $d^{d} \mathbf{r}:=d^{l} \mathbf{r}_{\|} d^{d-l} \mathbf{r}_{\perp}$. The properties of the initial disordered model are obtained in the limit $n \rightarrow 0$. Dimensional analysis of the action (16) yields the bare dimensions: $\left[\mathbf{r}_{\|}\right]=\mu^{-1 / 2},\left[\mathbf{r}_{\perp}\right]=\mu^{-1},[\psi]=[\bar{\psi}]=\mu^{(2 d-l-2) / 4}$ and $\left[\alpha_{j}\right]=\mu^{\delta}$, where $\mu$ is an arbitrary momentum scale and $\delta=2+l / 2-d$. Thus the upper critical dimension of the model (16) is $d_{c}=2+l / 2$ and disorder is (i) relevant for $\delta>0$, e.g. for $2 \mathrm{D}$ semi-Dirac fermions with $\delta=1 / 2$; (ii) marginally relevant for 2D Dirac fermions with $\delta=0$; (iii) irrelevant for $3 \mathrm{D}$ Weyl $(\delta=-1)$ and semi-Weyl fermions $(\delta=-1 / 2)$.

One-loop $R G$ with restricted disorder. We focus here on the case of $2 \mathrm{D}$ semi-Dirac fermions. To renormalize the model (16) one has to eliminate UV divergences in the loop expansion by introducing counter terms. The one-loop counterterms to the propagator and disorder are given by the diagrams shown in fig. 5 To one-loop order the $m$ and $c$ do not get corrected while $\Delta$ gets an additive shift similar to that in eq. (6). We use dimen-

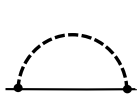

(a)

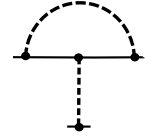

(b)

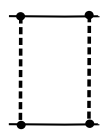

(c)

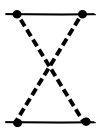

(d)
Fig. 5: The one-loop diagrams contributing to the propagator (a) and disorder renormalization (b)-(d).

sional regularization in which this shift vanishes [35. The diagrams (c) and (d) in fig. 5 can generate extra terms with new algebraic structures like $\gamma_{i} \gamma_{j} \gamma_{k} \otimes \gamma_{i} \gamma_{j} \gamma_{k}$ and so on. In $d=d_{c}-\delta$ dimensions they have to be treated as independent new interactions reflecting the fact that within dimensional regularization this theory is not multiplicatively renormalizable 39]. For the Dirac fermions this problem shows up to orders higher than one-loop and can be solved, for example, using a cutoff in strictly $2 \mathrm{D}$ so that the extra interactions do not arise. In contrast, for the semi-Dirac fermions the extra interactions infinitely proliferate in the renormalization already to the one-loop order. This cannot be cured by using a different regularization scheme since the upper critical dimension $\frac{5}{2}$ is non integer. To avoid this obstacle we restrict ourselves to the presence of a single type of disorder. It is easy to see that the diagrams (c) and (d) in fig. 5 with any type of disorder always contribute to $\alpha_{1} \equiv \alpha_{x}$. Thus we can derive a closed one-loop flow equation by considering a model with only the $\alpha_{x}$ type of disorder. The corresponding RG equations read

$$
-\mu \partial_{\mu} \ln \varepsilon=1+\alpha_{x}, \quad-\mu \partial_{\mu} \alpha_{x}=\delta \alpha_{x}-\alpha_{x}^{2},
$$

where we have included the value of the one-loop integral in redefinition of $\alpha_{x}$. Equations (17) possess a fixed point $\alpha_{x}^{*}=\delta$. Integrating them up to the scale corresponding to $\varepsilon \simeq 1$, we find $\varepsilon=\mu^{z}$ with $z=1+\alpha_{x}^{*}=1+\delta+O\left(\delta^{2}\right)$. The DOS behaves as $\rho \sim\langle\bar{\psi} \psi\rangle$, which leads to its scaling as $\rho \sim \mu^{3 / 2-z}$ or equivalently in terms of energy as

$$
\rho(\varepsilon) \sim \varepsilon^{(3 / 2-z) / z}=\varepsilon^{(1 / 2-\delta) /(1+\delta)+O\left(\delta^{2}\right)} .
$$

Hence we find that for $\delta=\frac{1}{2}$ the DOS exponent vanishes to one loop order suggesting a constant DOS at zero energy when only the $\alpha_{x}$ disorder is present. This result improves upon the critical behavior extracted from the SCBA analysis which was shown to be invalid at low energy. Whether the exponent vanishing is accidental or holds beyond the one-loop order remains an open question.

Full one-loop RG analysis. To analyze the scaling behavior of the full disordered 2D semi-Dirac fermions, we find it more technically tractable to consider an alternative generalized model given by the effective action

$$
\begin{gathered}
\mathcal{S}=\int d^{d} \mathbf{r}\left\{\sum_{a=1}^{n} \bar{\psi}_{a}\left[\sigma_{x}\left(\Delta-\frac{\nabla_{\|}^{2}}{2 m}\right)-i c \sigma_{y} \partial_{y}-i \varepsilon\right] \psi_{a}\right. \\
\left.-\frac{\pi c}{\sqrt{m}} \sum_{a, b=1}^{n} \sum_{j=0, x, y, z} \alpha_{j}\left[\bar{\psi}_{a}(\mathbf{r}) \sigma_{j} \psi_{a}(\mathbf{r})\right]\left[\bar{\psi}_{b}(\mathbf{r}) \sigma_{j} \psi_{b}(\mathbf{r})\right]\right\} .
\end{gathered}
$$


where $d^{d} \mathbf{r}:=d^{d-1} \mathbf{r}_{\|} d \mathbf{r}_{\perp}$. The advantage of this model is that it involves no infinite Clifford algebra. Dimensional analysis of this model suggests that its upper critical dimension is $d_{c}=3$. We can construct a perturbative in disorder RG around this dimension using expansion in a small parameter $\delta=(3-d) / 2$ similar to that done for the model (16). The corrections to energy and disorder strengths are given by the same diagrams shown in fig. (5). The one-loop RG equations read

$$
\begin{aligned}
& -\mu \partial_{\mu} \ln \varepsilon=1+\alpha_{0}+\alpha_{x}+\alpha_{y}+\alpha_{z}, \\
& -\mu \partial_{\mu} \alpha_{0}=\delta \alpha_{0}+4 \alpha_{0}\left(\alpha_{0}+2 \alpha_{x}+\alpha_{y}+\alpha_{z}\right)+4 \alpha_{x} \alpha_{z}, \\
& -\mu \partial_{\mu} \alpha_{x}=\delta \alpha_{x}+2\left(\alpha_{0}^{2}+\alpha_{x}^{2}+\alpha_{y}^{2}+\alpha_{z}^{2}\right)+4 \alpha_{0} \alpha_{z}, \\
& -\mu \partial_{\mu} \alpha_{y}=\delta \alpha_{y}+4 \alpha_{x} \alpha_{y}, \\
& -\mu \partial_{\mu} \alpha_{z}=\delta \alpha_{z}-4 \alpha_{z}\left(\alpha_{0}-2 \alpha_{x}-\alpha_{y}+\alpha_{z}\right)+4 \alpha_{0} \alpha_{x} .
\end{aligned}
$$

Unfortunately, the solutions of these RG equations always flow towards a strong disorder regime which describes Anderson localization, but is not within the reach of a weak disorder RG analysis. In this generalized model, we do no find a critical perturbative fixed point, as opposed to the model with only $\alpha_{x}$ disorder.

Conclusions. - We have studied the effect of disorder on the topological merging transition from a Dirac semi-metal to a band insulator in $2 \mathrm{D}$ systems. Using the SCBA we have found that disorder smears out the transition and an intermediate disordered semi-Dirac regime emerges. This phase is separated from the insulating phase by a sharp continuous transition while there is a crossover between this phase and disordered Dirac fermions regime. Exactly at the transition the SCBA suggests a power-law behavior of DOS with a nontrivial exponent $\frac{1}{3}$. We have also shown that the critical point can be perturbatively described using a weak disorder RG when only random fluctuations of $\Delta$ are present but is unfortunately inaccessible for the most general model. It would also be interesting to study the effect of disorder on other types of merging transitions [40.

$$
* * *
$$

We would like to thank G. Montambaux for inspiring and useful discussions. This work has been supported by the ANR project 2010-BLANC-041902 (IsoTop).

\section{REFERENCES}

[1] Geim A. K. and Novoselov K. S., Nature Materials, 6 (2007) 183.

[2] Castro Neto A. H. et al, Rev. Mod. Phys., 81 (2009) 109.

[3] Fu L., Kane C. L. and Mele E. J., Phys. Rev. Lett., 98 (2007) 106803.

[4] Moore J. E. and Balents L., Phys. Rev. B, 75 (2007) 121306(R).

[5] Hsien D. et al, Nature, 452 (2008) 970.
[6] Hancock J. N. et al, Phys. Rev. Lett., 107 (2011) 136803.

[7] Katayama S., Kobayashi A. and Suzumura Y. J. Phys. Soc. Jpn., 75 (2006) 054705.

[8] Kobayashi A. et al, J. Phys. Soc. Jpn., 76 (2007) 034711.

[9] Goerbig M.O. et al, Phys. Rev. B, 78 (2008) 045415.

[10] Nishine T., Kobayashi A. and Suzumura Y., J. Phys. Soc. Jpn., 79 (2010) 114715.

[11] Gomes K. K. et al, Nature, 483 (2012) 306.

[12] Wunsch B., Guinea F. and Sols F., New J. Phys., 10 (2008) 103027.

[13] Montambaux G. et al, Eur. Phys. J. B, 72 (2009) 509; Phys. Rev. B, 80 (2009) 153412.

[14] Dietl P., Piéchon F. and Montambaux G., Phys. Rev. Lett., 100 (2008) 236405.

[15] Volovik G. E., JETP Lett., 73 (2001) 162; The Universe in a Helium Droplet (Clarendon Press, Oxford) 2003.

[16] Lim L.-K., Fuchs J.-N. and Montambaux G., Phys. Rev. Lett., 108 (2012) 175303.

[17] Delplace P. and Montambaux G., Phys. Rev. B, 82 (2010) 035438.

[18] Tarruell L. et al, Nature, 483 (2012) 302.

[19] Rechtsman M. C. et al, arXiv:1211.5683

[20] Bellec M. et al, Phys. Rev. Lett., 110 (2013) 033902.

[21] Kobayashi A. et al, Phys. Rev. B, 84 (2011) 075450.

[22] Suzumura Y., Morinari T. and PiÉchon F., J. Phys. Soc. Jpn., 82 (2013) 023708.

[23] Ludwig A. W. W. et al, Phys. Rev. B, 50 (1994) 7526.

[24] Nersesyan A. A., Tsvelik A. M. and Wenger F., Nucl. Phys. B, 438 (1995) 561.

[25] Horovitz B. and Le Doussal P., Phys. Rev. B, 65 (2002) 125323

[26] Aleiner I. L. and Efetov K. B., Phys. Rev. Lett., 97 (2006) 236801.

[27] Ostrovsky P. M., Gornyi I. V. and Mirlin A. D., Phys. Rev. B, 74 (2006) 235443.

[28] Shon N.H. and Ando T., J. Phys. Soc. Jpn., 67 (1998) 2421.

[29] Khveshchenko D. V., Phys. Rev. B, 75 (2007) 241406(R); EPL, 82 (2008) 57008.

[30] Fedorenko A. A., Carpentier D. and Orignac E., Phys. Rev. B, 85 (2012) 125437.

[31] Dutreix C. et al, Phys. Rev. B, 87 (2013) 245413.

[32] Adroguer P., Carpentier D., Montambaux G. and ORIGNAC E., (unpublished).

[33] Lifshitz I. M., Sov. Phys. JETP, 11 (1960) 1130.

[34] Fukuzawa T., Koshino M. and Ando T., J. Phys. Soc. Jpn., 78 (2009) 094714.

[35] Dienl H. W. and Shpot M., Phys. Rev. B, 62 (2000) 12338.

[36] Banerjee S. and Pickett W. E., Phys. Rev. B, 86 (2012) 075124.

[37] Ziman J. M., Principles of the Theory of Solids (Cambridge University Press, Cambridge UK) 1972, p. 332

[38] Delplace P., Li J. and Carpentier D., EPL, 97 (2012) 67004 .

[39] Bennett J. F. and Gracey J. A., Nucl. Phys. B, 563 (1999) 390.

[40] VoloviK G. E., Springer Lecture Notes in Physics, 718 (2007) 31. 